\title{
Investigation of Dispersion and Performance Based on Ring Cavity by Birefringent Interleaver for DWDM Transmission Systems
}

\author{
Tsair-Chun Liang and Chun-Ting Chen \\ Graduate Institute of Electrical Engineering, National Kaohsiung First University of Science and Technology, Kaohsiung City 811, Taiwan \\ Correspondence should be addressed to Tsair-Chun Liang; tcliang@nkfust.edu.tw
}

Received 10 September 2013; Accepted 7 October 2013

Academic Editor: Teen-Hang Meen

Copyright (C) 2013 T.-C. Liang and C.-T. Chen. This is an open access article distributed under the Creative Commons Attribution License, which permits unrestricted use, distribution, and reproduction in any medium, provided the original work is properly cited.

\begin{abstract}
We theoretically investigate a $25 \mathrm{GHz}$ multichannel filter based on ring cavity birefringent optical interleaver for dense wavelength division multiplexing (DWDM) transmission systems. The simulation tool used in this work is the Advanced System Analysis Program (ASAP) optical modeling software. We improve the dispersion performance by employing $\lambda / 6$ and $\lambda / 4$ wave plates as birefringent compensators for interleavers. The new structure exhibits a high performance with nearly zero ripple, a channel isolation greater than $102 \mathrm{~dB}$, and a passband utilization of $86 \%$ within the C-band. The research results illustrate that our modified scheme can improve the dispersion of more than $76.6 \%$ in comparison with the previous studies of optical interleaver with birefringent crystal and ring cavity structures.
\end{abstract}

\section{Introduction}

In the recent years, with the rapid growth of internet and the maturity of multimedia conferencing, dense wavelength division multiplexing (DWDM) $[1,2]$ has emerged as a vital component for optical fiber networks. And how to increase the number of channels is an important issue [3]. One way to increase the channels number is to widen the usable wavelength bandwidth in low-loss region of the used single-mode fiber $[4,5]$. Another way to increase the channels number is to narrow the channel spacing. Several techniques have been engaged in DWDM systems with channel spacing of less than $0.8 \mathrm{~nm}[6,7]$. A spectral interleaver is capable of separating a set of channels into two sets at twice the channel spacing $[8,9]$. An optical interleaver has been verified as an effective technique in increasing channel counts by doubling or quadrupling the number of optical channels when the channel spacing is in the range of $0.2 \mathrm{~nm}(25 \mathrm{GHz})$. Nevertheless, the greatest shortcoming of conventional interleavers is an inferior dispersion.

In this paper, we improve the dispersion performance by employing $\lambda / 6$ and $\lambda / 4$ wave plates as birefringent compensators for Sagnac-interferometer-based flat-top birefringent optical interleaver employing a ring cavity as a phase-shift element, which was proposed by Lee et al. [10]. The simulation tool used in this work is the Advanced System Analysis Program (ASAP) optical modeling software [11]. And the interleaver design model is configured based on the actual component parameters.

\section{Configuration of the Proposed Birefringent Interleaver}

Figure 1 depicts the configuration of ring cavity birefringent interleaver. For this scheme, an optical circulator, a polarization beam splitter (PBS), two birefringent crystals $\left(\mathrm{YVO}_{4}\right.$ has the length of $30 \mathrm{~mm}$ ), a triangular-shape prism (transmission 91.4\%; refraction index 1.6), and four highly reflective mirrors (reflectivity 99.8\%) are used. In this structure, we proposed two $\lambda / 4$ wave plates and two $\lambda / 6$ wave plates as dispersion compensators employed in the ring cavity to ensure excellent flat-top spectral passband. The $\mathrm{YVO}_{4}$ birefringent crystal is used for appropriate retardance of interference. The $\lambda / 6$ and $\lambda / 4$ wave plates are engaged to rotate the beam polarization state by 30 and 45 degrees, respectively. 


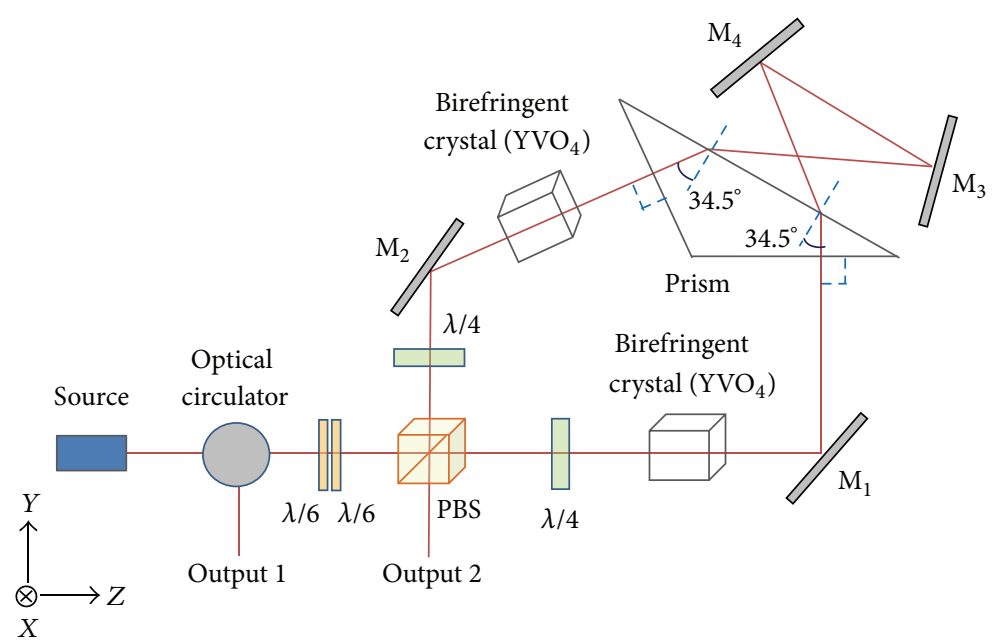

FIGURE 1: Configuration of the proposed ring cavity birefringent interleaver.

The input signal of unpolarized light in the 1530-1565 nm wavelength range (C-band) with a channel spacing of $0.2 \mathrm{~nm}$ is considered. When the input signal was transmitted through the PBS, the s-component propagates along the loop in the clockwise direction and the p-component propagates along the loop in the counterclockwise direction. As a result, the beams inside the birefringent crystals consist of both the ordinary wave and the extraordinary wave with equal amplitudes. As the beams propagate inside the birefringent crystals, a phase retardation exists between these two waves at the end of the crystals. These beams, consisting of both ordinary wave (o-ray) and extraordinary wave (e-ray), are then directed toward the ring cavity which is configured by a prism and two mirrors $\left(\mathrm{M}_{3}\right.$ and $\left.\mathrm{M}_{4}\right)$.

In the two birefringent crystals, the ordinary wave corresponds to s-wave, while the extraordinary wave corresponds to $\mathrm{p}$-wave. The prism interface of the ring cavity exhibits different Fresnel reflectivities for these two polarization components ( $s$ and $\mathrm{p}$ ) of the beam. Due to the different reflectivities, these two polarization components experience different phase shifts upon transmitting (or reflecting) through the ring cavity. Through the ring cavity, these two components of the beam incur further phase retardation from the birefringent crystal before they are mixed and recombined by the PBS.

\section{Computer Simulation}

The air-prism interface is aligned perpendicularwise to the light beams, as shown in Figure 1. And the prism is cut into a triangular shape to provide an appropriate angle of incidence so that the desired Fresnel reflectivities, $R_{\mathrm{o}}$ and $R_{\mathrm{e}}$, are obtained. $R_{\mathrm{e}}$ and $R_{\mathrm{o}}$ are the reflectivities of the airprism interface for the e-ray and the o-ray. In this work, the optimum incident angle is near $34.5^{\circ}$, and, at this angle of incidence, the reflectivities are $R_{\mathrm{o}}=17.01 \%$ and $R_{\mathrm{e}}=8.39 \%$. The normalized intensity of one of the output ports can be expressed as follows $[12,13]$ :

$$
I=\frac{I_{0}}{2}\left[I_{0}+\cos \left(\frac{2 \pi}{\lambda}\left(n_{\mathrm{e}}-n_{\mathrm{o}}\right) L+\left(\phi_{\mathrm{e}}-\phi_{\mathrm{o}}\right)\right)\right],
$$

where $I_{0}$ is the intensity of the unpolarized incident beam, $L$ is the length of the two birefringent crystals, $\phi_{\mathrm{e}}$ and $\phi_{\mathrm{o}}$ are the phase shifts of the beam for the e-ray and the oray, respectively, upon reflection from the ring cavity, and $\Delta n$ $\left(=n_{\mathrm{e}}-n_{\mathrm{o}}\right)$ is the refractive index difference of $n_{\mathrm{e}}$ and $n_{\mathrm{o}}$. Chromatic dispersion compensation [14] is the most deserving in our study because the dispersion is the parameter which restricts the transmission distance of DWDM systems. The polarization azimuth angle of the birefringent crystal $[15,16]$ is obtained by $\lambda / 4$ wave plates $\left(\lambda / 4\right.$ at $\left.45^{\circ}\right)$ and $\lambda / 6$ wave plates $\left(\lambda / 6\right.$ at $\left.30^{\circ}\right)$. Then, the output group delay after compensation can be viewed as the average group delay from two modes, $\tau(\omega)=\left[\tau_{\mathrm{e}}(\omega)+\tau_{\mathrm{o}}(\omega)\right] / 2$, and can be shown as in (2), where $\omega=2 \pi \mathrm{c} / \lambda$ is the optical angular frequency, $T=L_{R} / \mathrm{c}$ is the round-trip time in the ring cavity, $L_{R}$ is the round-trip optical path of the ring cavity, and $d_{1}$ and $d_{2}$ are the round-trip phase shifts of $\lambda / 4$ wave plates and $\lambda / 6$ wave plates, respectively. The group velocity dispersion (GVD) is given by $D(\lambda)=\mathrm{d} \tau / \mathrm{d} \lambda$ (ps/nm) and can be obtained as in (3). Consider the following:

$$
\begin{aligned}
& \tau(\omega) \\
& =\frac{T}{2}\left\{\left(1-R_{\mathrm{o}}\right) \times\left(1+R_{\mathrm{o}}-2 \sqrt{R_{\mathrm{o}}} \cos \left(\left(\frac{2 \pi}{\lambda}\right) L_{R}\right)\right)^{-1}\right. \\
& +\left(1-\left(\frac{R_{\mathrm{e}}}{\left(d_{1}^{2}+d_{2}^{2}\right)}\right)\right) \\
& \times\left(1+\left(\frac{R_{\mathrm{e}}}{\left(d_{1}^{2}+d_{2}^{2}\right)}\right)-2\left(\frac{R_{\mathrm{e}}}{\left(d_{1}^{2}+d_{2}^{2}\right)}\right)^{1 / 2}\right. \\
& \left.\left.\quad \times \cos \left(\left(\frac{2 \pi}{\lambda}\right) L_{R}\right)\right)^{-1}\right\}
\end{aligned}
$$

$D(\lambda)$

$$
=\frac{2 \pi L_{R} T \sin \left((2 \pi / \lambda) L_{R}\right)}{\lambda^{2}}
$$




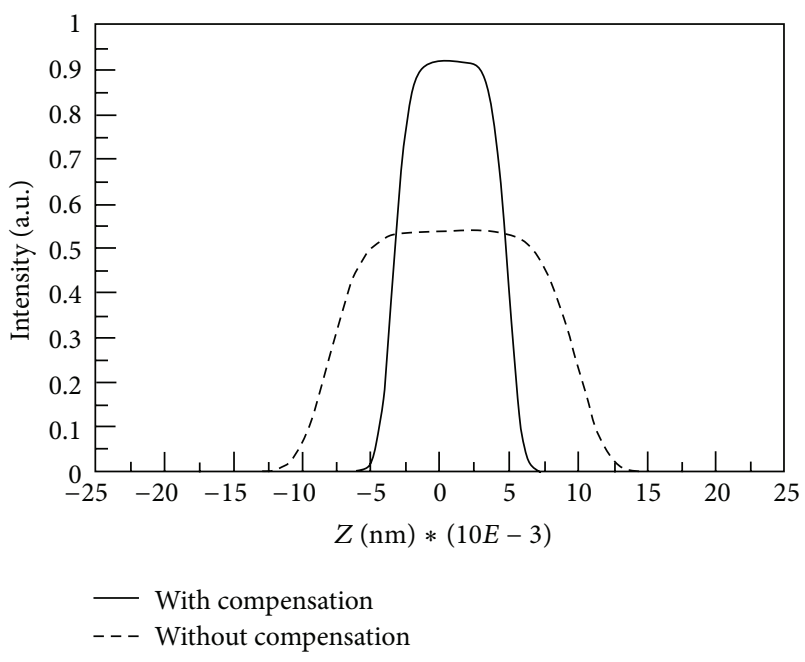

(a)

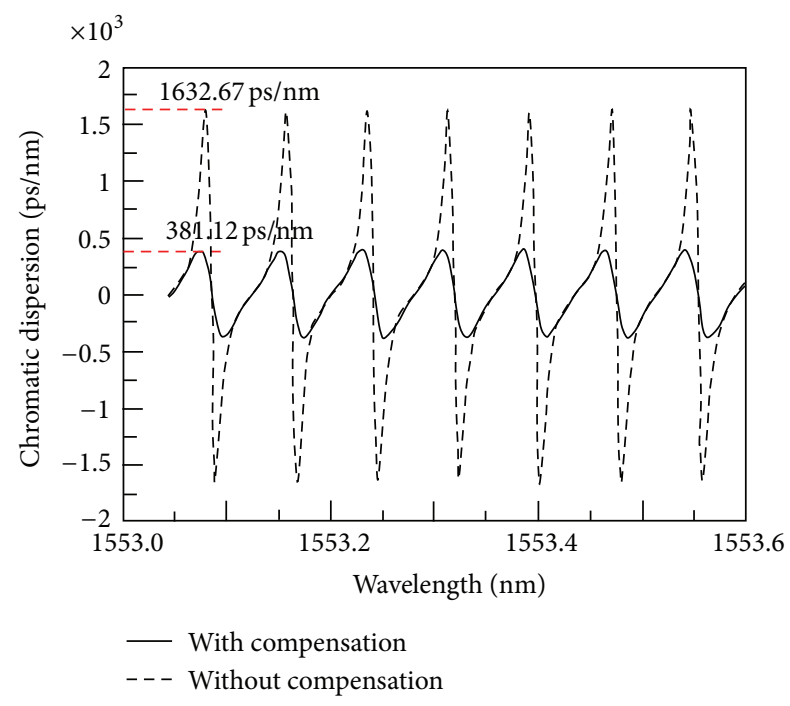

(b)

FIGURE 2: The optical spectral (a) signal intensity of one of the output ports and (b) chromatic dispersion characteristics of partial C-band, with and without compensation.

$$
\begin{gathered}
\times\left\{\left(R_{\mathrm{o}}\right)^{1 / 2}\left(1-R_{\mathrm{o}}\right) \times\left(\left[1+R_{\mathrm{o}}-2\left(R_{\mathrm{o}}\right)^{1 / 2}\right.\right.\right. \\
\left.\left.\times \cos \left(\left(\frac{2 \pi}{\lambda}\right) L_{R}\right)\right]^{2}\right)^{-1} \\
+\left(\frac{\left(\mathrm{R}_{e}\right)}{\left(d_{1}^{2}+d_{2}^{2}\right)}\right)^{1 / 2}\left(1-\left(\frac{R_{\mathrm{e}}}{\left(d_{1}^{2}+d_{2}^{2}\right)}\right)\right) \\
\times\left(\left[1+\left(\frac{\left(R_{\mathrm{e}}\right)}{\left(d_{1}^{2}+d_{2}^{2}\right)}\right)-\frac{2\left(R_{\mathrm{e}}\right)}{\left(d_{1}^{2}+d_{2}^{2}\right)^{1 / 2}}\right.\right. \\
\left.\left.\left.\quad \times \cos \left(\left(\frac{2 \pi}{\lambda}\right) L_{R}\right)\right]^{2}\right)^{-1}\right\}
\end{gathered}
$$

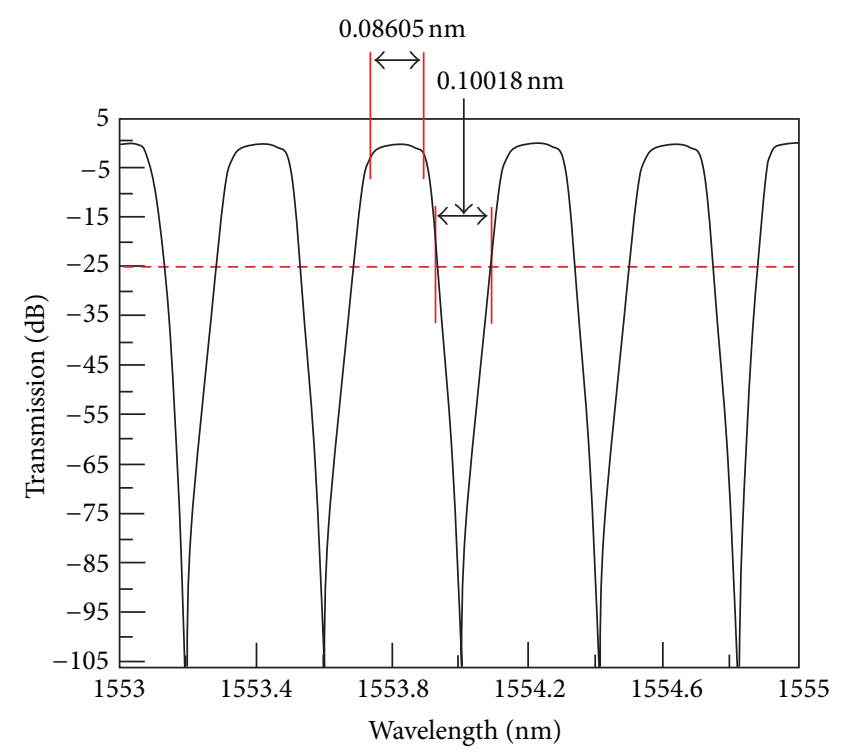

(a)

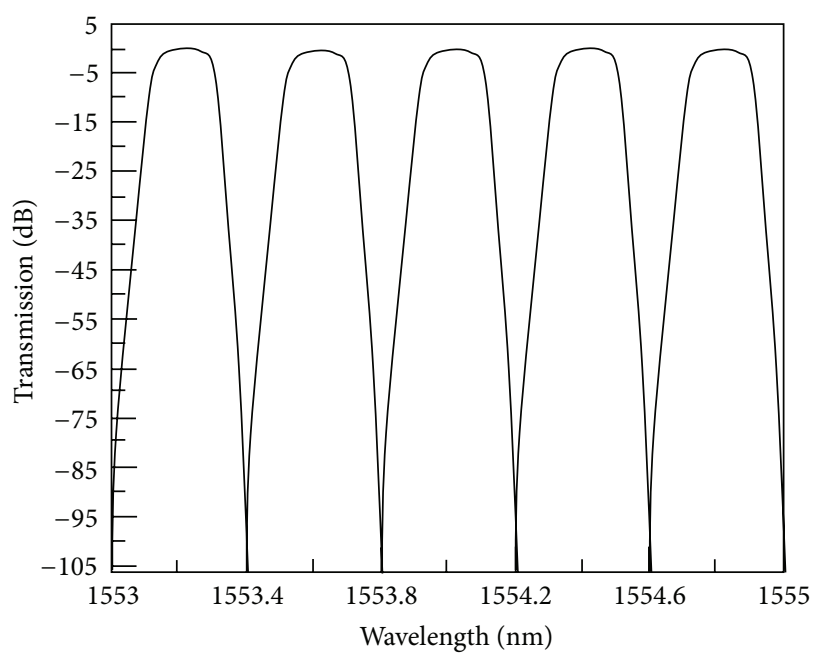

(b)

FIGURE 3: The relative transmission power of partial C-band: (a) output port 1 (odd channels) and (b) output port 2 (even channels).

According to (1) and (3), calculated by the simulation software ASAP, we can get normalized intensity of the output channels and their chromatic dispersion. Figure 2(a) shows the calculated spectral output power of one channel. The optical intensities of with- and without-compensation schemes are 0.91 a.u. and 0.553 a.u., respectively. Figure 2(b) shows the chromatic dispersion of partial C-band comparison between with- and without-compensation schemes of $25 \mathrm{GHz}$ channel spacing. The research results illustrate that our modified scheme can improve the dispersion of more than $76.6 \%(=(1632.67-381.12) / 1632.67)$ in comparison. The channel isolation of the interleaver with compensators is greater than $102 \mathrm{~dB}$, and the calculated results of the stopband and channel isolation of a $25 \mathrm{GHz}$ channel spacing application are shown in Figure 3 . The $25 \mathrm{~dB}$ stopband was found to be $0.10018 \mathrm{~nm}$, the $0.5 \mathrm{~dB}$ wide passband was found 


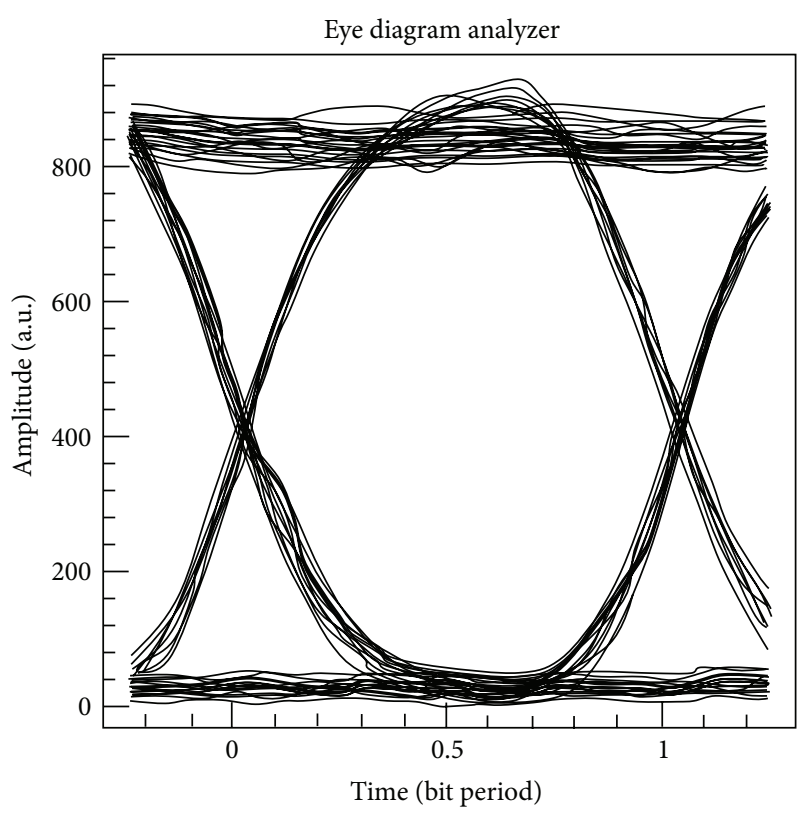

(a)

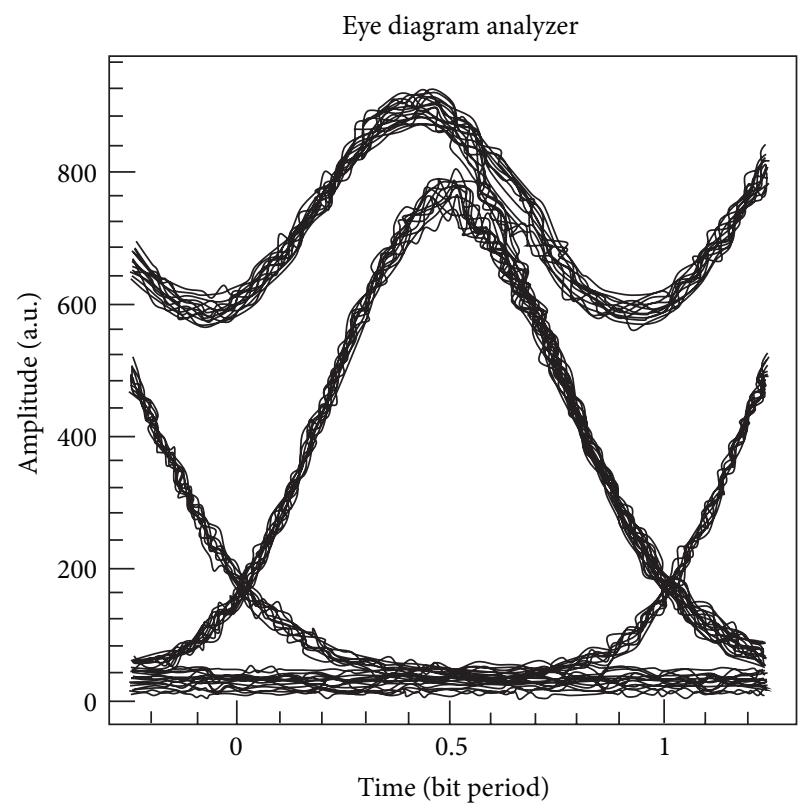

(b)

FIGURE 4: The eye diagrams by PRBS $2^{31}-1$ for $10 \mathrm{~Gb} / \mathrm{s}$ application: (a) with compensation and (b) without compensation.

to be $0.08605 \mathrm{~nm}$, and the passband utilization was $86 \%$ $(=0.08605 \mathrm{~nm} / 0.10018 \mathrm{~nm})$ within the C-band. Figure 4 shows the eye diagrams for $10 \mathrm{~Gb} / \mathrm{s}$ application of with- and without-compensation schemes by pseudorandom binary sequence (PRBS) $\left(2^{31}-1\right)$.

\section{Conclusions}

We have investigated the characteristics of a flat-top $25 \mathrm{GHz}$ optical interleaver based on ring cavity architecture with and without dispersion compensation elements. We found that the ring cavity birefringent interleaver with two $\lambda / 4$ wave plates and two $\lambda / 6$ wave plates as birefringent compensators exhibited a $0.5 \mathrm{~dB}$ passband larger than $10.75 \mathrm{GHz}$ $(0.08605 \mathrm{~nm})$, a $25 \mathrm{~dB}$ stopband greater than $12.52 \mathrm{GHz}$ $(0.10018 \mathrm{~nm})$, and a channel isolation higher than $102 \mathrm{~dB}$. The benefit of this interleaver is that it utilizes the Fresnel principle to achieve precise reflectivities. Unlike dielectric mirrors with thin-film coatings, the reflectivities of the Fresnel reflection are insensitive to wavelength variations in the transmission band. The uniform reflectivities are essential to ensure the same performance over the entire C-band. In particular, the novel interleaver can simultaneously produce the excellent performance of chromatic dispersion that achieved an improvement of $76.6 \%$ when compared to the currently available interleaver without wave plates as birefringent compensators, which was proposed by Lee et al. [10]. This modified interleaver may find important applications in DWDM systems and transmission networks.

\section{Acknowledgment}

This work was partly supported by the National Science Council of the Republic of China, under Contracts nos. NSC 101-2221-E-327-032 and NSC 102-2622-E-327-005-CC3.

\section{References}

[1] R. Saunders, "Coherent DWDM technology for high speed optical communications," Optical Fiber Technology, vol. 17, no. 5, pp. 445-451, 2011.

[2] Y. C. Chi, C. J. Lin, S. Y. Lin, and G. R. Lin, "The reuse of downstream carrier data erased by self-feedback SOA for bidirectional DWDM-PON transmission," Journal of Lightwave Technology, vol. 30, pp. 3096-4643, 2012.

[3] R. Casellas, R. Munoz, J. M. Fabrega et al., "GMPLS/PCE control of flexi-grid DWDM optical networks using CO-OFDM transmission," Journal of Optical Communications and Networking, vol. 4, pp. B1-B10, 2012.

[4] T.-C. Liang, Y.-K. Chen, J.-H. Su et al., "Optimum configuration and design of 1480-nm pumped L-band gain-flattened EDFA using conventional erbium-doped fiber," Optics Communications, vol. 183, no. 1-4, pp. 51-63, 2000.

[5] M. Matsuura, M. Taguchi, and N. Kishi, "S, C, L-band signal transmission using a widely tunable optical clock generator," Optics Communications, vol. 281, no. 21, pp. 5423-5428, 2008.

[6] S. Zirak-Gharamaleki, "Narrowband optical filter design for DWDM communication applications based on Generalized Aperiodic Thue-Morse structures," Optics Communications, vol. 284, no. 2, pp. 579-584, 2011.

[7] Z. Zhou, S. L. Xiao, T. Qi, P. Q. Li, M. H. Bi, and W. S. Hu, "25GHz-spaced DWDM-PON with mitigated rayleigh backscattering and back-reflection effects," IEEE Photonics Journal, vol. 5, no. 4, Article ID 7901407, 2013.

[8] G. R. Bhatt, R. Sharma, U. Karthik, and B. K. Das, "Dispersionfree SOI interleaver for DWDM applications," Journal of Lightwave Technology, vol. 30, no. 1, pp. 140-146, 2012.

[9] N. Kumar, M. R. Shenoy, and B. P. Pal, "Flattop all-fiber wavelength interleaver for DWDM transmission: design analysis, parameter optimization, fabrication and characterization 
recipe," Optics Communications, vol. 281, no. 20, pp. 5156-5164, 2008.

[10] C.-W. Lee, R. Wang, P. Yeh, and W.-H. Cheng, "Sagnac interferometer based flat-top birefringent interleaver," Optics Express, vol. 14, no. 11, pp. 4636-4643, 2006.

[11] ASAP (Advanced Systems Analysis Program), Breault Research Organization, Tucson, Ariz, USA.

[12] S. Cao, J. Chen, J. N. Damask et al., "Interleaver technology: comparisons and applications requirements," Journal of Lightwave Technology, vol. 22, no. 1, pp. 281-289, 2004.

[13] B. B. Dingel and M. Izutsu, "Multifunction optical filter with a Michelson-Gires-Tournois interferometer for wavelength-division-multiplexed network system applications," Optics Letters, vol. 23, no. 14, pp. 1099-1101, 1998.

[14] L. Wei and J. W. Y. Lit, "Design optimization of flattop interleaver and its dispersion compensation," Optics Express, vol. 15, no. 10, pp. 6439-6457, 2007.

[15] J. Zhang, L. Liu, and Y. Zhou, "Novel and simple approach for designing lattice-form interleaver filter," Optics Express, vol. 11, pp. 2217-2224, 2003.

[16] M. Oguma, T. Kitoh, Y. Inoue et al., "Compact and low-loss interleave filter employing lattice-form structure and silicabased waveguide," Journal of Lightwave Technology, vol. 22, no. 3, pp. 895-902, 2004. 


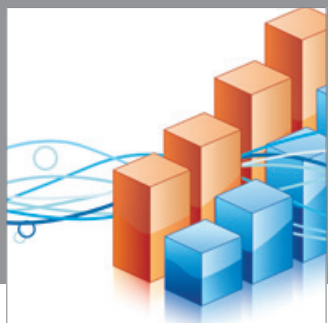

Advances in

Operations Research

mansans

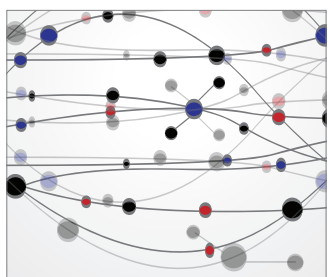

The Scientific World Journal
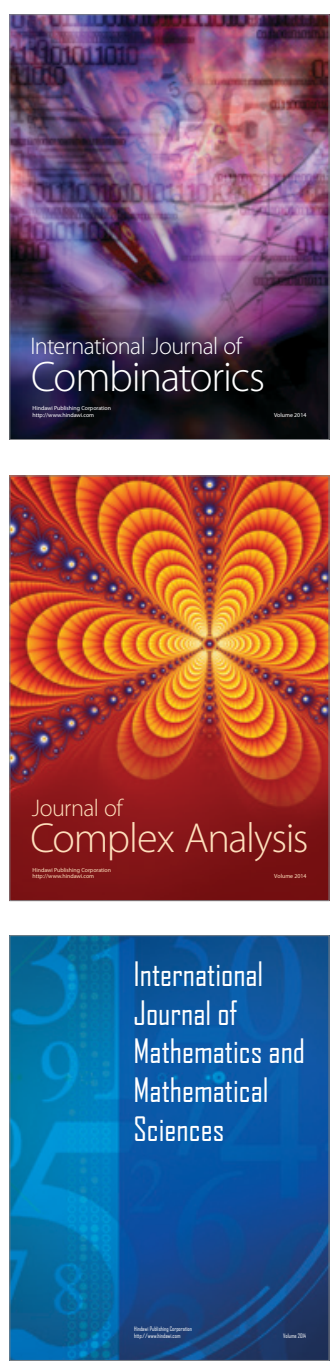
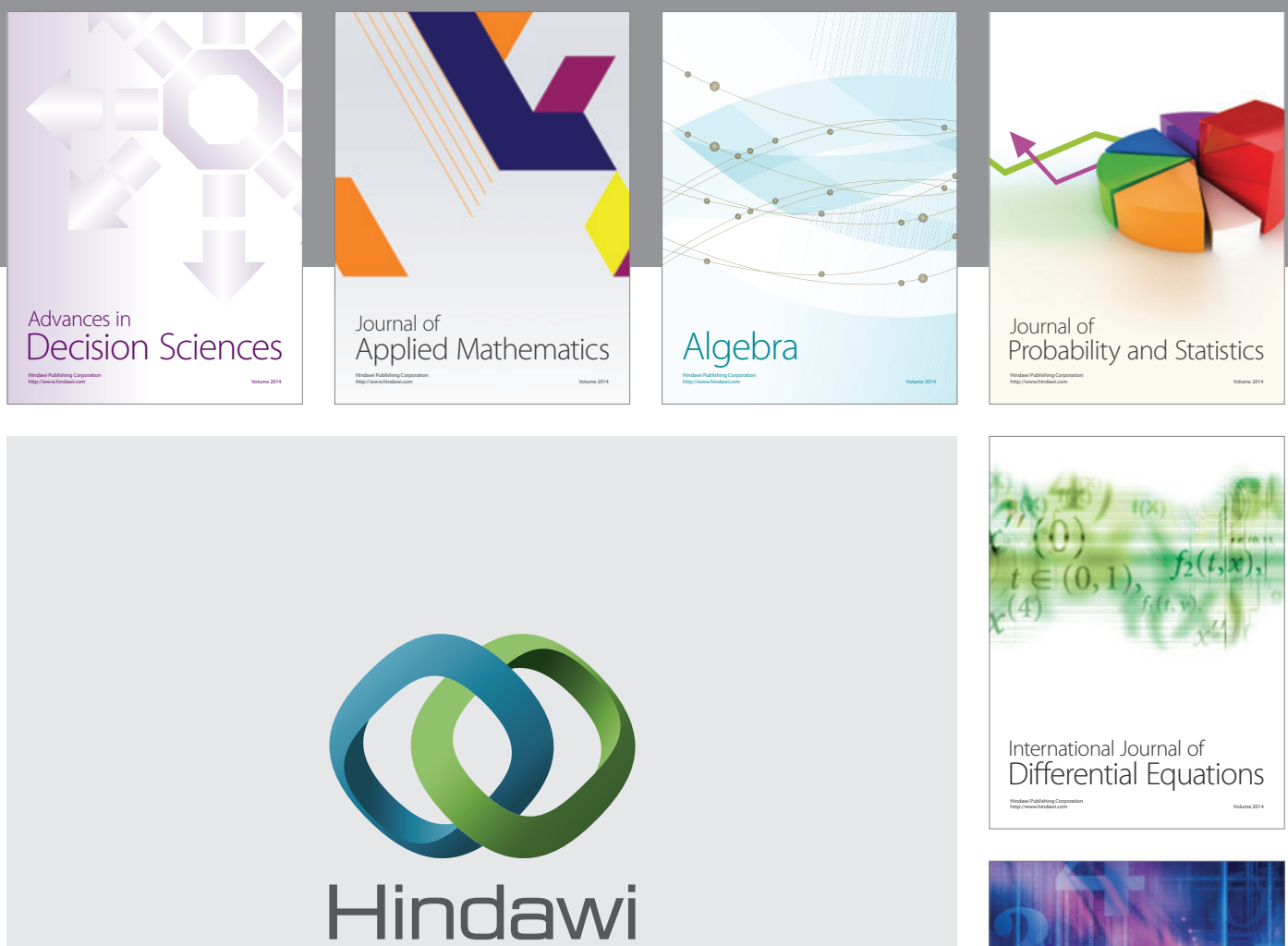

Submit your manuscripts at http://www.hindawi.com
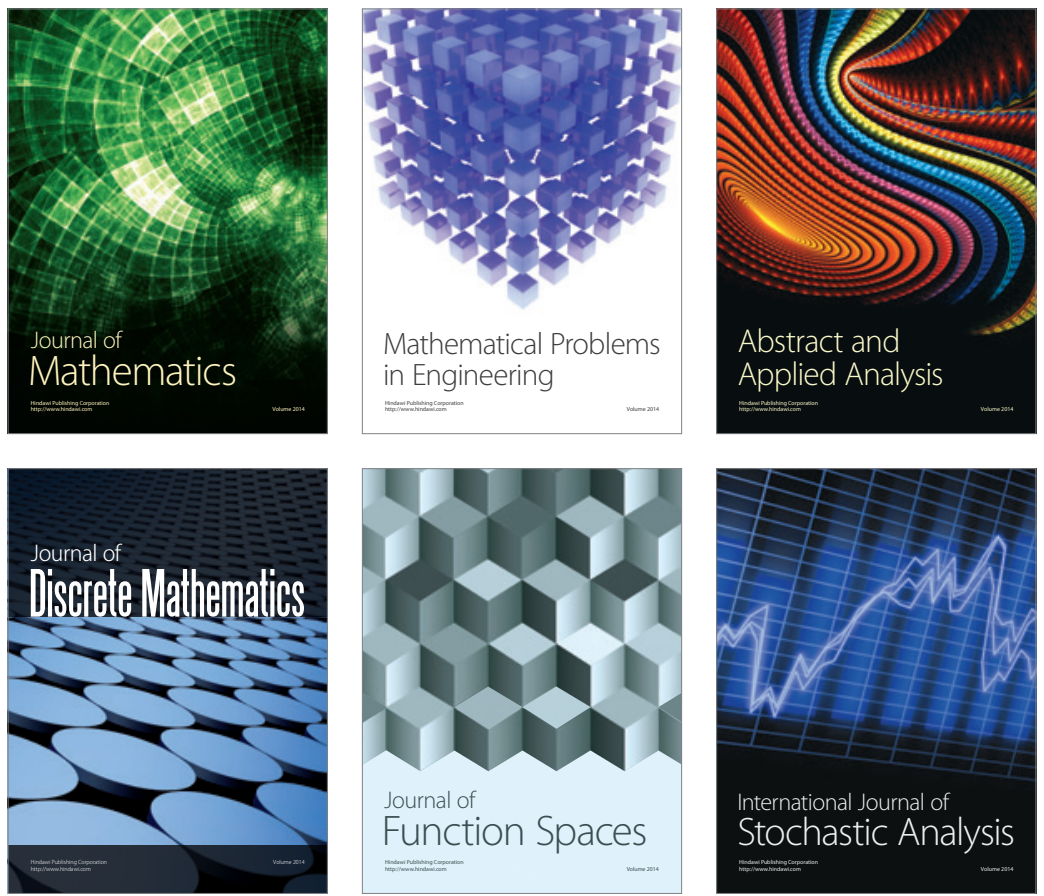

Journal of

Function Spaces

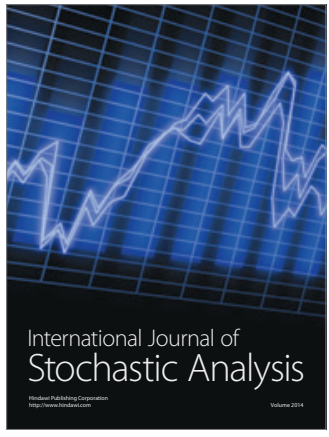

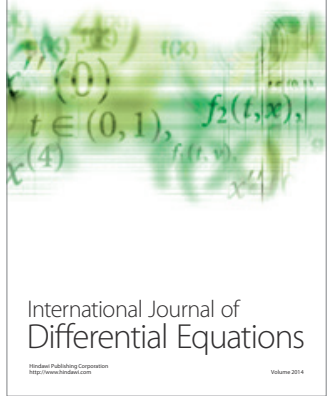
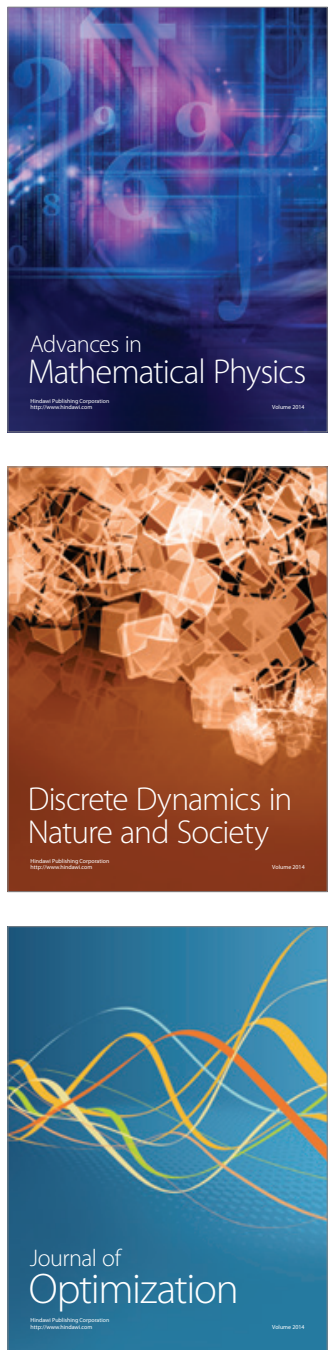